\section{CONGRESO DE LA}

\section{SOCIEDAD ESPAÑOLA DE HISTORIA DE LA CIENCIA (SEHC)}

La principal doctrina del empirismo lógico relativa a la historiografía de la ciencia se infería directamente de la manida separación contextual en virtud de la cual se establecía una distinción fundamental entre el desarrollo histórico-sociológico de la ciencia ("contexto de descubrimiento") y las reconstrucciones lógico-metodológicas de los logros científicos («contexto de justificación»). Consecuentemente, quedaba meridianamente determinada la línea de demarcación que definía los campos de actuación académicos de los historiadores y filósofos de la ciencia, de tal modo que para estos últimos resultaban irrelevantes las anomalías de la ciencia efectiva respecto al modelo de racionalidad científica propuesto.

Los trabajos de Hanson, Toulmin y Kuhn a partir de los últimos años de la década de los 50, señalan las primeras críticas al empirismo lógico mediante el recurso a la ciencia real sustentado en gran parte en el conocimiento de la historia de la ciencia. Así, un primer ejemplo del impacto de los estudios históricos en las teorías lógicas y epistemológicas de la filosofía de la ciencia se materializa en la tesis conclusiva de Kuhn según la cual no pueden justificarse los cambios de paradigma en base a razones empíricas o lógicas.

La nueva actitud de los filósofos de la ciencia frente a la historia se tradujo, en el mundo anglosajón preferentemente, en la proliferación de programas, departamentos y congresos destinados, por una parte, a la clarificación de la relación existente entre ambas disciplinas y, por otra, a la actualización de los conocimientos históricos.

La celebración del III Congreso de la SEHC que tuvo lugar en San Sebastián y Vitoria del 1 al 6 de octubre debe interpretarse, desde esta perspectiva, como un acontecimiento de indudable relevancia en nuestro mapa cultural. Tras los dos primeros Congresos -celebrados en 1978 (Santiago Garma (ed.), I Congreso de la Sociedad Española de Historia de las Ciencias, Madrid 1980) y 1982 (M. Hormigón (ed.), Actas del II Congreso de la Sociedad Española de Historia de las Ciencias, Zaragoza 1984)- este III Congreso reunió cerca de doscientos estudiosos del campo de la filosofía y la historia de la ciencia, cifra elocuentemente indicativa del interés por los temas afrontados en el encuentro. Se leyeron nueve ponencias y el número de comunicaciones sobrepasó el centenar, cubriéndose las siguientes áreas: ciencia y técnica en Euskadi; la ciencia en la época de Leibniz y Newton (1650-1750); historia de las instituciones científicas en España: historia de las aplicaciones de las ciencias formales a las ciencias humanas, sociales y naturales; y una última sección de tema libre. Tuvieron lugar además diversas actividades como excursiones, visitas guiadas, etc. y una mesa redonda sobre "Ciencia y técnica en Euskadi». Pudieron darse a conocer, en suma, un buen número de trabajos interesantes y novedosos que contribuirán a aportar sin duda conoci. mientos útiles para la historiografía de la ciencia.

Las limitaciones de esta nota nos impiden hacernos eco de las comunicaciones, por lo que habrá de sernos suficiente con reseñar brevemente el tema de las conferencias.

Leandro Silván, catedrático de Ciencias Quími* cas y académico de las Reales Academias de la His. toria (Madrid) y de Ciencias y Artes (Barcelona) disertó sobre «la Medicina y la Alquimia. Evolución histórica y relaciones mutuas". A lo largo de su documentada exposición, el profesor Silván indicó las relaciones mutuas existentes entre la medicina y la ciencia hermética desde la paleomedicina y las actividades prealquímicas, analizando no tanto la ciencia hermética que se ocupa de perfeccionar las diversas formas de la materia, y en particular los metales a partir de transmutaciones precisas, cuanto la que persigue el logro de la perfección total de las energías vitàles a fin de mantener incólume la salud corporal y prolongar así de manera indefinida la existencia de los seres vivos. Es esta segunda función de la alquimia, derivada de una concepción antropológica de la materia, la que establece una relación fundamental entre ella y la medicina a lo largo de la historia hasta su ocaso con el advenimiento de una nueva disciplina, la química, asentada sobre hipótesis distintas a las suyas (teoría del flogisto de Stahl, Boyle, etc.).

La ponencia de Olivier Baulny, de la Universi" dad de Pau, prosiguió temáticamente (si bien su participación en el programa fue anterior) la conferencia del profesor Silván. Su tema: «L'image de 
la matière et la naissance de la chimie, de Nicolas Lémery à Lavoisiem. Analizó el origen de la química glosando la figura de Lémery en torno a su concepción de la materia e indicó la aportación capital de Lavoisier cuya explicación del fenómeno de la combustión y de la oxidación-reducción acabó con la teoría del flogisto y sentó las bases de la química de nuestros días.

Tres ponencias tuvieron como centro a LeibnizNewton: las desarrolladas por Víctor Gómez-Pin, Ivo Schneider y Eberhard Knobloch. Este último, catedrático de Historia de la Ciencia de la Universidad de Berlín, tituló su conferencia «Leib. niz and his mathematical heritagen. En realidad, en su calidad de director de la edición de los escritos matemáticos de Leibniz de la Academia de Ciencias de Berlín, expuso mediante copias de los manuscritos leibnizianos las contradicciones presentes en los textos originales, así como las dificultades, tanto técnicas como simbólicas, inheren" tes a la transcripción de tales textos. Dificultades que han debido ser superadas para la preparación del volumen I de los escritos matemáticos de Leibniz.

Ivo Schneider, catedrático de Historia de la Ciencia de la Universidad de Múnich, realizó un brillante recorrido histórico sobre las ideas algorítmicas en la matemática de los siglos XVI al XVuI. En su «Algoritmic thinking in Mathematics from the 16th to 18 th centuries" analizó las raíces del pensamiento algorítmico que culmina en el "calculus» y el análisis del siglo XvnI. Ivo Schneider expuso de manera clara, coherente y precisa la interconexión existente entre tales raíces, desde el origen del término "algoritmo" hasta la creencia en la posibilidad de una explicación comprehensiva del universo que incluyera el mundo de los seres vivos, pasando por un cúmulo de factores que contribuyeron a crear las condiciones de posibilidad del «algorithmic thinking» tales como los métodos de enseñanza de la aritmética elemental de la época, la función de los símbolos tras la «invención" de la imprenta en el siglo XV, la necesidad de controlar la práctica ar. tesanal a partir de órdenes estrictas expresadas me. diante instrucciones secuenciales, el «universalismon concebido como la posibilidad de existencia de un método susceptible de resolver todos los problemas de un dominio dado y, finalmen- te, las funciones pedagógicas derivadas de instrumentos científicos como el círculo proporcional.

Señaló Ivo Schneider que el escepticismo de Descartes respecto a los algoritmos y sus posibilidades radicaba tanto en su creencia de que la explicación mecánica tan sólo es plausible en una porción de las actividades humanas, cuanto en la inconsistencia, como lo muestra su polémica con Fermat, del método algorítmico con su original método, único susceptible de ofrecer, según Descartes, la posibilidad de alcanzar nuevos conocimientos. La resistencia cartesiana no pudo impedir el desarrollo de diversos métodos y reglas algorítmicas (Fermat, los «indivisibles» de Cavalieri, John Wallis) que darían paso al cálculo infinitesimal y, ya en el siglo XVIII, al análisis de Bernouilli y Euler, de indudable carácter algorítmico. El declive del formalismo durante el siglo siguiente se debió, según el ponente, tanto al creciente número de problemas surgidos en su seno cuanto al agotamiento de sus posibilidades y, sobre todo, a un tercer factor relacionado con la justificación social del desarrollo de las reglas algorítmicas, a saber, la reforma del sistema educacional a partir de la revolución francesa hizo decrecer el número de matemáticos dependientes de secuencias de instrucciones cuyos principios quedaban inexplicados, lo cual contribuyó a generar un profundo cambio en el carácter de la matemática durante las décadas iniciales del XIX.

«Categoría de cualidad y concepto de variable en el cálculo diferencial» fue la ponencia que sirvió de colofón antes de la clausura oficial del Congreso. En ella, Víctor Gómez.Pin, de la Univer. sidad de País Vasco, destacó la trascendencia del cálculo diferencial tanto para la matemática como para la filosofía, definiéndolo como paradig. ma de un saber no abstracto y denunciando «la imposibilidad de acceso a tal saber que las actuales (y escandalosas para un Leibniz o un Newton) condiciones de división del trabajo intelec. tual suponen». Desde este punto de vista analizó de modo riguroso el recurso, utilizado por Newton, de abstraer los infinitesimales de órdenes su. periores al demostrar el lema según el cual si $A B$ son las cantidades «en mutación" constitutivas de un rectángulo y ab los respectivos "momentos" o velocidades de mutación, entonces el «momen- 
ton o velocidad de mutación en el rectángulo $A B$ sería Ab-aB.

Tras el acto inaugural, el primero en tomar la palabra entre los profesores invitados fue C. Ulises Moulines, catedrático de Filosofía de la Ciencia de la Universidad de Bielefeld y uno de los exponentes más relevantes, junto a Sneed y Stegmüller, de lo que se ha dado en llamar la ucon. cepción estructural de las teorías científicas». En su esperada conferencia, "Filosofía de la ciencia - historiografía de la ciencia: ¿dos caras de la misma medalla?", retomó ideas ya expuestas con anterioridad", definiendo a ambas disciplinas como pertenecientes a las ciencias de la cultura con un objeto de estudio común: el fenómeno cultural que denominamos «ciencia". ¿Cuáles son, pues, las diferencias que las separan? Podríamos considerar, al respecto, las siguientes dicotomías presentes en la literatura sobre la cuestión: la historiografía de la ciencia se atiene a los hechos, en tanto que la filosofía de la ciencia es pura especulación teórica; la primera es (básicamente) descriptiva, la segunda es (básicamente) normativa; siguiendo el criterio de Windelband, la primera sería idiográfica, mientras que la segunda sería nomotética; $y$, en fin, la primera es diacrónica, la segunda sincrónica.

El profesor Moulines rechazó como insatisfactorios estos pares dicotómicos porque: el primero de ellos obvia la idea formulada entre otros por Lakatos según la cual «la historia (es decir, la historiografía) sin alguna preconcepción teórica es imposbile», del mismo modo que la filosofía de la ciencia se atiene (cada vez más) a la forma y al contenido de la ciencia real; la dicotomía descriptivo/normativo es consecuencia del «tercer dogma» del empirismo (todo discurso con sentido es o bien descriptivo o bien normativo), dogma falso que impide la correcta apreciación de las dos disciplinas como esencialmente «interpretativas» o ureconstructivas» ${ }^{2}$; la tercera dicotomía

' C. Ulises Movines, «On How the Distinction Between History and Philosophy of Science Should Not Be Drawn", Erkenntris, vol. 19, no 1-3, mayo 1983.

${ }^{2}$ En el sentido expuesto por primera vez por W. STrGmoLLER en "A Combined Approach to the Dynamics of Theories", Theory and Decision, 9, 1978. (Hay traducción castellana de D. Ribes, «Planteamiento combinado de la dinámica de teorias", P. Feyerabend, G. RADNitZKy y otros, Estruc. tura y desarrollo de la ciencia, Alianza, Madrid 1984, pp. 261-262.) es insatisfactoria porque, en tanto que ciencias de la cultura, tanto la filosofía como la historiografía de la ciencia tratan con «fenómenos irrepetibles», lo cual no impide su interés común por lograr un cierto nivel de "abstracción generalizadora»; la dicotomía diacrónico/sincrónico no es válida sino para los estadios iniciales de nuestras disciplinas, pues la realidad es que ambos aspectos tienden a combinarse sistemáticamente desde las dos perspectivas como lo muestran los trabajos de la escuela estructuralista de Sneed-Stegmüller y el estilo creado en la historiografía por Kuhn.

Sin embargo, en opinión de Moulines, a pesar de las constataciones últimas, existen elementos que nos hacen pensar en la oportunidad del mantenimiento de la independencia de ambas perspectivas, por cuanto se trata de dos interpretaciones de la ciencia de signo distinto: una, la historiográfica, de orden "tipológico", busca identificar «tipos» en el sentido de Max Weber; la otra, filosófica, de orden "morfológico», va a la búsqueda de estructuras. En todo caso, concluyó, la distinción no es tajante sino "probablemente una cuestión de grado".

La falta de espacio nos obliga a una breve mención de las tres ponencias de carácter institucional. Ernesto García Camarero, presidente de la SEHC, disertó sobre "La transición de la Junta al Consejo Superior de Investigaciones Científicas durante la guerra civil española», analizando el origen del actual CSIC desde su precedente inmediato, la Junta para Ampliación de Estudios e Investigaciones Científicas (JAE). La ponencia del profesor Juan José Saldaña, presidente de la Sociedad Latinoamericana de Historia de las Ciencias y Tecnología (México, UNAM), «La introducción de la óptica ondulatoria a principios del siglo Xxx: el papel de las instituciones", centro la atención preferente en la función beligerante de las instituciones francesas en la introducción de la óptica ondulatoria en aquel país. La ponencia de Jesús Arpal Poblador, de la Universidad del País Vasco, "La formación científico-técnica en la sociedad estamental: a propósito del Seminario de Vergara», constituyó una variación al programa y en ella se analizó el proceso de producción científico-técnica en una sociedad estamental en contraposición con la sociedad de clases y la sociedad industrial. 
Las jorm: das del congreso sirvieron de marco, además, para la realización de la asamblea de la SEHC en la que se nombró una nueva Junta Directiva, al tiempo que se fijó en Valladolid la sede del próximo congreso a celebrar en 1986. En suma, el IIT Congreso de la SEHC marcó la con- solidación, no exenta de errores ciertamente, de un foro válido para la promoción de intercam. bios sugerentes en el dominio de la historia (y la fliosofía) de la ciencia.

Andoni lbarra
IV SEMINARIO DE HISTORIA DE LA FILO. SOFÍA ESPAÑOLA

Organitzat pel Departamento de Historia de la Filosofía de la Universidad de Salamanca i per l'Instituto de Ciencias de la Educación de la mateixa Universitat, entre els dies 24 i 28 de setembre es va celebrar la quarta edició del Seminario de Historia de la Filosofía Española.

En aquestes pàgines ja s'ha parlat d'aquest seminari que se celebra cada dos anys a Salamanca. En aquest sentit, i com que les tres edicions anteriors han estat recollides en forma de libre, no cal que entrem en moltes presentacions del seu caràcter general. Només diguem que el seminari es consolida cap a la seva finalitat principal, que no és altra que la de contribuir a la comunicació entre aquells que es dediquen als diversos aspectes de la història de la filosofia espanyola - potser per a nosaltres resultaria més còmode dir-ho tor en plural: històries de les filosofies espanyoles, és un dir-, deixant de banda, -i això no és poc important - caure en especulacions que interroguen per les dubtoses essències de les filosofies de les nacions, i prescindint, per tant, de les velles polèmiques del passàt.

Aquest any, les activitats han tingut una secció principal dedicada a les filosofies hispanoamericanes. Les diverses ponències -entre d'altres de N.R. Orringer, J.L. Martínez-Gómez, A.M. Ro- dríguez Cruz i W. J. Kilgore- han servit per mos" trar la dispersió del tema, la necessitat d'enfocar" lo cap a una síntesi -encara que només sigui una abstracta idea de context $-i$ l'elevat grau d'especialització que comporta cadascun dels seus continguts. Si més no, no s'ha de perdre de vista que el període a estudiar és molt ampli -comença amb la colonització espanyola d'Amèrica- $\mathrm{i}$ la diversitat de països no pot ser més gran.

Un altre apartat del seminari ha estat dedicat a la visió que es té de la filosofia espanyola a Fran. ça $\mathrm{i}$ a Itàlia. Les ponències van córrer a càrrec d'Alain Guy i de Pietro Prini, respectivament. Pensant en una futura ampliació d'aquesta secció, que no deixa de tenir el seu interès, no estaria de més tenir present que la visió que es té de la filosofia espanyola des d'altres països es pot enfocar des d'una perspectiva molt menys optimista, de manera que l'anàlisi estarà molt més apropada a la realitat.

D'una altra secció, la dedicada a diverses qüiestions de la filosofia espanyola actual, podem destacar tres ponències que són representatives de tres maneres d'aproximar-se a la historia de la filosofia, si més no en aquest seminari: la de Miguel Cruz Hernández sobre Zubiri - un model del que pot ser una bona aportació de les vivències personals a l'estudi d'un determinat filòsof - , la de José Chamizo sobre l'estat actual de la filosofia del llenguatge a Espanya -un exemple de treball ben documentat i de síntesi- i la d'Alfredo San- 